Research Article

\title{
Synthesis of a New Type of Trans-Decalin Vitamin D Analogue through a Dyotropic Ring Expansion
}

\author{
Hugo Santalla (iD, Uxía Gómez-Bouzó, Irene Sánchez-Sanz, and Yagamare Fall \\ Departamento de Química Orgánica, Facultad de Química and Instituto de Investigación Biomédica (IBI), University of Vigo, \\ Campus Lagoas de Marcosende, Vigo 36310, Spain
}

Correspondence should be addressed to Hugo Santalla; hsantalla@uvigo.es

Received 10 June 2021; Accepted 8 September 2021; Published 7 October 2021

Academic Editor: Arturo Espinosa Ferao

Copyright $(92021$ Hugo Santalla et al. This is an open access article distributed under the Creative Commons Attribution License, which permits unrestricted use, distribution, and reproduction in any medium, provided the original work is properly cited.

A new vitamin D analogue with a trans-fused decalin as the CD-ring system and containing a sulphur atom in the side chain has been synthesized in our research group. The obtention of this analogue is based on a recently discovered transformation of hydrindane cores into decalins through a dyotropic ring expansion in very mild conditions.

\section{Introduction}

Calcitriol 1 (Figure 1) or 1 $\alpha, 25$-dihydroxyvitamin $\mathrm{D}_{3}$, the biologically active form of vitamin $\mathrm{D}_{3}$, has a potential application as a therapy agent in the treatment of different pathologies related to antiproliferative and cell differentiation activity [1-8]; however, it shows as the main limitation its calcemic effects. Actually, there is great interest in the obtention of new calcitriol analogues with less calcemic toxicity.

Facing this goal, an interesting research area is the synthesis of nonsteroidal analogues where the CD fragment is modified, substituting the natural trans-fused hydrindane bicyclic system for a monocyclic moiety such as five- $[9,10]$ or six-membered rings [11-14].

In fact, one of the most used modifications is the replacement of the trans-hydrindane core for a trans-decalin core (Figure 1).

Decalins are bicyclic carbon frameworks that can be found in numerous polyterpenoid and steroid natural products with interesting biological activity [15-23]. In our laboratory, we recently reported a very useful stereospecific methodology to convert hydrindane moieties into decalin derivatives through a new type of dyotropic reaction involving a mesylate group in mild conditions with high yields (Scheme 1) [24].
These decalin cores have been recently used as the building block to obtain interesting functionalized decalins which represents a challenging task for organic chemists such as chiral thioethers [25].

Thus, we can access disulphide 5 by displacement of mesylate 4 with potassium thioacetate. Alkylation of 5 with an electrophile $[26,27]$ using sodium borohydride afforded (between others) compound 6, our precursor in the synthesis of a new trans-decalin vitamin D analogue (Scheme 2 ).

From chiral building block 6 as the initial substrate, we report on easy synthesis of a novel type of trans-decalin vitamin $\mathrm{D}$ analogue with a sulphur atom as the linker between the side chain and CD fragment as depicted in Scheme 3 (compound 7).

\section{Results and Discussion}

The synthesis is based on a convergent route which involves the construction of Grundmann's ketone 8 followed by Wittig-Horner coupling using phosphine oxide 9 to afford the triene system, as shown in the retrosynthetic scheme (Scheme 3).

First, compound 6 was deprotected using a TBAF solution $1.0 \mathrm{M}$ in THF and heating at $65^{\circ} \mathrm{C}$ several days to transform both silyl ethers into hydroxyl groups, affording diol 10 with 97\% yield as shown in Scheme 4. 


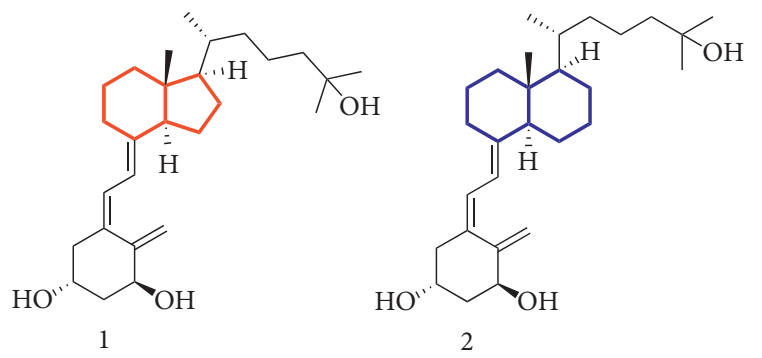

Figure 1: Structure of calcitriol 1 and trans-decalin vitamin D analogue 2.
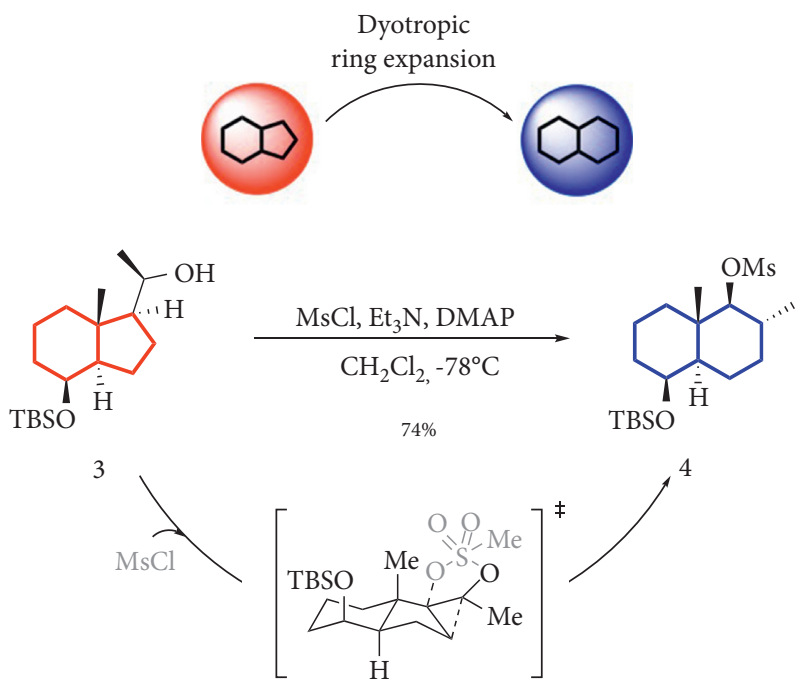

SCHEME 1: Ring expansion of 3 via the dyotropic ring expansion to afford decalin 4 .

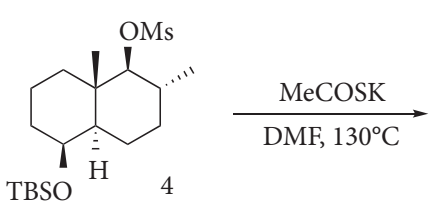

$87 \%$

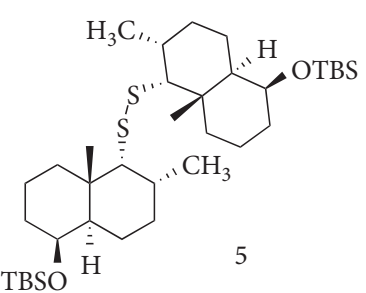<smiles>CCOC(C)(C)CCS[C@H]1[C@@H](C)CC[C@H]2[C@@H](O)CCC[C@]12C</smiles>

1) $\mathrm{NaOH}, \mathrm{NaBH}_{4}$

2) $\mathrm{EtOH}$<smiles>CC(C)(CCBr)O[SbH2]</smiles>

$75 \%$

Scheme 2: Obtention of key intermediate 6 from mesylate 4.

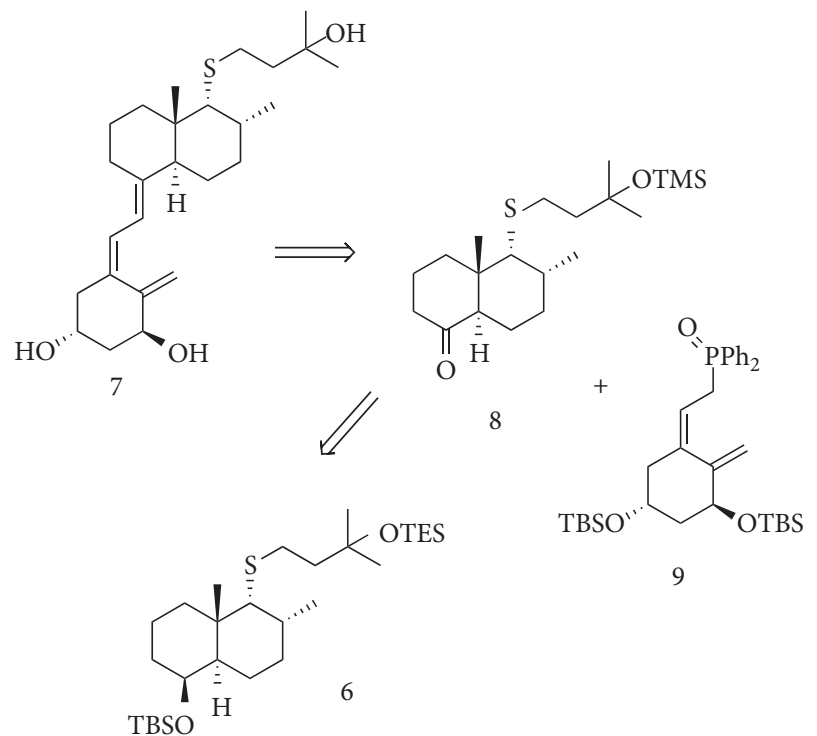

Scheme 3: Retrosynthetic scheme for the obtention of a new transdecalin vitamin $\mathrm{D}$ analogue with a sulphur atom.
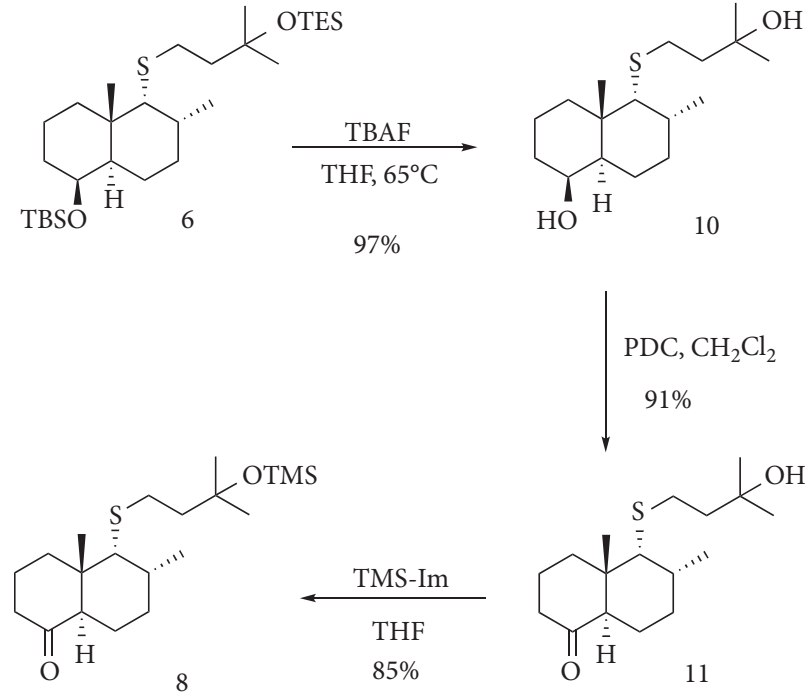

Scheme 4: Synthetic sequence to obtain Grundmann's ketone 8.

An oxidant such as PDC was used to convert diol 10 into Grundmann's ketone derivative 11 with $91 \%$ yield which was protected with TMS-imidazole in THF, obtaining Grundmann's ketone synthon 8 with $85 \%$ yield.

Compound 8 was converted into 12 via the Wittig-Horner reaction $[28,29]$ with phosphine oxide 9 with $55 \%$ yield. Removal of the TBS-protecting groups in 12 gave analogue 7 with 99\% yield as shown in Scheme 5. 


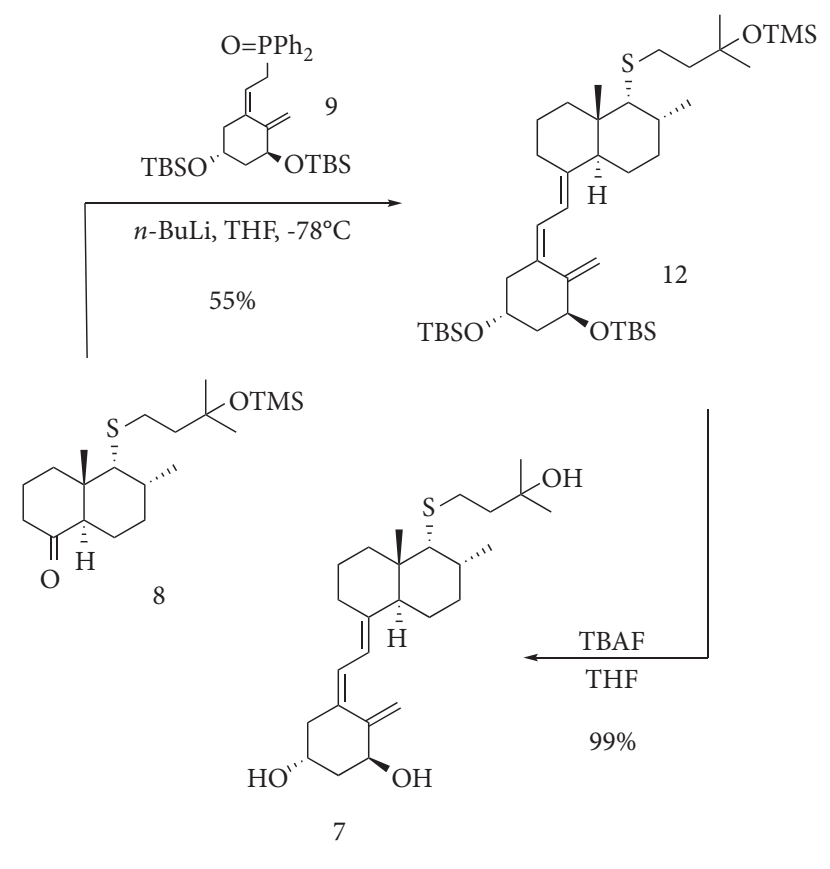

Scheme 5: Obtention of final analogue 7.

\section{Conclusions}

In conclusion, we have demonstrated how this dyotropic expansion reaction to convert a hydrindane into a decalin scaffold under very mild conditions can be a powerful tool to an easy access to vitamin D analogues with a trans-decalin core in its structure as the CD fragment. Work is now in progress towards the synthesis of new similar analogues as well as their biological evaluation.

\section{Experimental Section}

For experimental procedures, characterization data and copies of ${ }^{1} \mathrm{H}$ and ${ }^{13} \mathrm{C}$ spectra of all compounds were used; see the Supplementary Materials (available here).

General: solvents were purified and dried by standard procedures before use. Melting points were uncorrected. ${ }^{1} \mathrm{H}$ NMR and ${ }^{13} \mathrm{C}$ NMR spectra were recorded with a Bruker ARX-400 spectrometer $\left(400 \mathrm{MHz}\right.$ for ${ }^{1} \mathrm{H} \quad \mathrm{NMR}$ and 100.61 MHz for ${ }^{13} \mathrm{C}$ NMR) using TMS as the internal standard (chemical shifts in $\delta$ values, $J$ in $\mathrm{Hz}$ ). Flash chromatography (FC) was performed on silica gel (Merck 60, 230-400 mesh); analytical TLC was performed on plates precoated with silica gel (Merck 60 F254, $0.25 \mathrm{~mm}$ ); mass spectra (FAB and EI) were recorded using Fisons VG, and electron spray ionization (ESI-MS) spectroscopy was recorded using Bruker FTMS APEXIII. Melting points were obtained in open capillary tubes and were not corrected. Optical rotations were obtained using a Jasco P-2000 polarimeter. IR spectra were recorded with a Jasco FT/I(R)6100 spectrophotometer.

Synthesis of $(1 S, 4 \mathrm{a} S, 5 R, 6 R, 8 \mathrm{a} R)-5-((3-$ hydroxy-3-methylbutyl)thio)-4a,6-dimethyldecahydronaphthalen-1-ol (10): TBAF 1.0M solution in THF $(0.92 \mathrm{ml}, 0.92 \mathrm{mmol})$ was added to another solution of $6(122 \mathrm{mg}, 0.23 \mathrm{mmol})$ in THF $(4 \mathrm{ml})$, and the mixture was stirred at $65^{\circ} \mathrm{C}$ for 3 days. After that time, 4 equivalents of TBAF were added, and the mixture was stirred in these conditions for another 3 days. After that time, the reaction was concentrated under reduced pressure. The residue was purified by column chromatography with EtOAc/hexane (30\%) as the eluent, affording $10(67 \mathrm{mg}$, 97\%) as colourless oil; Rf: 0.40 (50\% EtOAc/hexane). IR (ATR, $\mathrm{cm}^{-1}$ ): 3434, 3385, 2963, 2922, 2865, 1458, 1377, 1240, 915. $[\alpha]^{23}{ }_{\mathrm{D}}=+21.71\left(\right.$ c $\left.1.00, \mathrm{CHCl}_{3}\right) .{ }^{1} \mathrm{H}-\mathrm{NMR}\left(\mathrm{CDCl}_{3}, \delta\right)$ : $3.83(1 \mathrm{H}, \mathrm{d}, J=2.6 \mathrm{~Hz}, \mathrm{H}-1), 2.58\left(2 \mathrm{H}, \mathrm{m}, \mathrm{H}-1^{\prime}\right), 2.25(2 \mathrm{H}$, m), $2.12(1 \mathrm{H}, \mathrm{d}, J=3.1 \mathrm{~Hz}, \mathrm{H}-5), 1.79(5 \mathrm{H}, \mathrm{m}), 1.63(3 \mathrm{H}, \mathrm{m})$, $1.48(3 \mathrm{H}, \mathrm{m}), 1.27(2 \mathrm{H}, \mathrm{m}), 1.25\left(6 \mathrm{H}, \mathrm{s}, \mathrm{CH}_{3}-4^{\prime}\right), 1.22(3 \mathrm{H}, \mathrm{s}$, $\left.\mathrm{CH}_{3}-10\right), 1.17(1 \mathrm{H}, \mathrm{m}), 1.06\left(3 \mathrm{H}, \mathrm{d}, J=6.6 \mathrm{~Hz}, \mathrm{CH}_{3}-9\right) \mathrm{ppm}$. ${ }^{13} \mathrm{C}-\mathrm{NMR}\left(\mathrm{CDCl}_{3}, \delta\right): 71.8(\mathrm{CH}-1), 70.8\left(\mathrm{C}-3^{\prime}\right), 67.7$ (CH-5), $43.6\left(\mathrm{CH}_{2}\right), 41.4(\mathrm{CH}), 39.6(\mathrm{C}-8 \mathrm{a}), 36.7\left(\mathrm{CH}_{2}\right), 33.7\left(\mathrm{CH}_{2}\right)$, $31.2(\mathrm{CH}), 31.0\left(\mathrm{CH}_{2}\right), 30.5\left(\mathrm{CH}_{2}\right), 29.4\left(\mathrm{CH}_{3}-4^{\prime}\right), 29.3\left(\mathrm{CH}_{3}-\right.$ $\left.4^{\prime}\right), 25.7\left(\mathrm{CH}_{2}\right), 21.6\left(\mathrm{CH}_{3}-10\right), 21.0\left(\mathrm{CH}_{3}-9\right), 16.8\left(\mathrm{CH}_{2}\right)$ ppm. MS (ESI) ([m/z], \%): $283.20\left([\mathrm{M}-\mathrm{OH}]^{+}, 100\right), 301.22$ $\left([\mathrm{M}+1]^{+}, 55\right)$. HRMS (ESI): 301.2196 calculated for $\mathrm{C}_{17} \mathrm{H}_{33} \mathrm{O}_{2} \mathrm{~S}$ and found 301.2191 .

Synthesis of $(4 \mathrm{a} S, 5 R, 6 R, 8 \mathrm{a} R)-5-((3-$ hydroxy-3-methylbutyl)thio)-4a,6-dimethyloctahydronaphthalen-1(2H)-one (11): PDC (245 mg, $0.65 \mathrm{mmol})$ was added to a solution of alcohol 10 (65 mg, $0.21 \mathrm{mmol})$ in $\mathrm{CH}_{2} \mathrm{Cl}_{2}(3 \mathrm{ml})$, and the mixture was stirred at room temperature for 3 hours. Then, it was filtered, and the solvent was evaporated. The residue was purified by column chromatography with EtOAc/hexane (50\%) as the eluent, affording $11(58 \mathrm{mg}, 91 \%)$ as colourless oil; Rf: 0.34 (50\% EtOAc/hexane). IR (ATR, $\left.\mathrm{cm}^{-1}\right)$ : 3503, 2963, 2931, 2853,1708, 1455, 1366, 1248, 1039, 838. $[\alpha]$ ${ }_{\mathrm{D}}^{22}=+7.95\left(\mathrm{c} 1.00, \mathrm{CHCl}_{3}\right) .{ }^{1} \mathrm{H}-\mathrm{NMR}\left(\mathrm{CDCl}_{3}, \delta\right): 2.68(2 \mathrm{H}$, m, H-1' $), 2.59(1 \mathrm{H}, \mathrm{m}), 2.50(1 \mathrm{H}, \mathrm{dd}, J=11.8 / 4.0 \mathrm{~Hz}), 2.32$ $(3 \mathrm{H}, \mathrm{m}), 2.17(1 \mathrm{H}, \mathrm{m}), 2.01(1 \mathrm{H}, \mathrm{m}), 1.91(1 \mathrm{H}, \mathrm{m}), 1.79(2 \mathrm{H}$, ddd, $J=9.3 / 6.6 / 2.2 \mathrm{~Hz}), 1.52(5 \mathrm{H}, \mathrm{m}), 1.31(1 \mathrm{H}, \mathrm{m}), 1.26(6 \mathrm{H}$, s, $\left.\mathrm{CH}_{3}-4^{\prime}\right), 1.07\left(3 \mathrm{H}, \mathrm{d}, J=6.5 \mathrm{~Hz}, \mathrm{CH}_{3}-9\right), 0.96\left(3 \mathrm{H}, \mathrm{s}, \mathrm{CH}_{3^{-}}\right.$ 10) ppm. ${ }^{13} \mathrm{C}-\mathrm{NMR}\left(\mathrm{CDCl}_{3}, \delta\right): 213.4(\mathrm{C}=\mathrm{O}), 70.7\left(\mathrm{C}-3^{\prime}\right)$, 65.0 (CH-8a), $51.3(\mathrm{CH}-5), 45.4(\mathrm{C}-4 \mathrm{a}), 43.7\left(\mathrm{CH}_{2}\right), 41.3$ $\left(\mathrm{CH}_{2}\right), 36.2\left(\mathrm{CH}_{2}\right), 30.9(\mathrm{CH}), 30.8\left(\mathrm{CH}_{2}\right), 29.4\left(\mathrm{CH}_{3}-4^{\prime}\right)$, $29.3\left(\mathrm{CH}_{3}-4^{\prime}\right), 28.9\left(\mathrm{CH}_{2}\right), 22.6\left(\mathrm{CH}_{2}\right), 20.7\left(\mathrm{CH}_{3}-9\right), 20.7$ $\left(\mathrm{CH}_{2}\right), 18.9\left(\mathrm{CH}_{3}-10\right)$ ppm. MS (ESI) ([m/z], \%): 179.15 (100), $298.20\left([\mathrm{M}]^{+}, 19\right)$. HRMS (ESI): 298.1967 calculated for $\mathrm{C}_{17} \mathrm{H}_{30} \mathrm{O}_{2} \mathrm{~S}$ and found 298.1974 .

Synthesis of $(4 \mathrm{a} S, 5 R, 6 R, 8 \mathrm{a} R)-4 \mathrm{a}, 6$-dimethyl-5-((3methyl-3-((trimethylsilyl)oxy)butyl)thio)octahydronaphthalen-1(2H)-one (8): to a solution of ketone $11(60 \mathrm{mg}$, $0.2 \mathrm{mmol})$ in THF $(3 \mathrm{~mL})$, TMS-Im $(300 \mu \mathrm{L}, 2.0 \mathrm{mmol})$ was added, and the mixture was stirred at room temperature for 3 days. Then, it was quenched with $\mathrm{H}_{2} \mathrm{O}(5 \mathrm{~mL})$, and the mixture was extracted with EtOAc $(2 \times 10 \mathrm{~mL})$. The combined organic phases were dried over $\mathrm{Na}_{2} \mathrm{SO}_{4}$, filtered, and concentrated to give a residue which was chromatographed on silica gel using EtOAc/hexane (2\%) as the eluent, affording compound $8(63 \mathrm{mg}, 85 \%)$ as colourless oil; Rf: 0.55 (20\% EtOAc/hexane). IR (ATR, $\left.\mathrm{cm}^{-1}\right): 2963,2930$, $2852,1708,1455,1367,1250,1038,838 .[\alpha]_{\mathrm{D}}^{23}=+17.68$ (c $\left.1.00, \mathrm{CHCl}_{3}\right) .{ }^{1} \mathrm{H}-\mathrm{NMR}\left(\mathrm{CDCl}_{3}, \delta\right): 2.75(1 \mathrm{H}, \mathrm{td}, J=13.4 /$ $4.8 \mathrm{~Hz}), 2.57(3 \mathrm{H}, \mathrm{m}), 2.31(3 \mathrm{H}, \mathrm{m}), 2.16(1 \mathrm{H}, \mathrm{m}), 1.97(2 \mathrm{H}$, $\mathrm{m}), 1.72(2 \mathrm{H}, \mathrm{ddd}, J=9.4 / 6.4 / 2.0 \mathrm{~Hz}), 1.62(1 \mathrm{H}, \mathrm{m}), 1.51(3 \mathrm{H}$, m), $1.31(1 \mathrm{H}, \mathrm{m}), 1.24\left(6 \mathrm{H}, \mathrm{s}, \mathrm{CH}_{3}-4^{\prime}\right), 1.07(3 \mathrm{H}, \mathrm{d}, J=6.4 \mathrm{~Hz}$, 
$\left.\mathrm{CH}_{3}-9\right), 0.96\left(3 \mathrm{H}, \mathrm{s}, \mathrm{CH}_{3}-10\right)$ ppm. ${ }^{13} \mathrm{C}-\mathrm{NMR}\left(\mathrm{CDCl}_{3}, \delta\right)$ : $213.8(\mathrm{C}=\mathrm{O}), 73.4\left(\mathrm{C}-3^{\prime}\right), 64.3(\mathrm{CH}-8 \mathrm{a}), 51.3(\mathrm{CH}-5), 45.5$ (C-4a), $45.0\left(\mathrm{CH}_{2}\right), 41.3\left(\mathrm{CH}_{2}\right), 36.1\left(\mathrm{CH}_{2}\right), 30.8(\mathrm{CH}), 30.5$ $\left(\mathrm{CH}_{2}\right), 29.9\left(\mathrm{CH}_{3}-4^{\prime}\right), 29.8\left(\mathrm{CH}_{3}-4^{\prime}\right), 28.9\left(\mathrm{CH}_{2}\right), 22.7\left(\mathrm{CH}_{2}\right)$, $20.7\left(\mathrm{CH}_{3}-9\right), 20.7\left(\mathrm{CH}_{2}\right), 18.9\left(\mathrm{CH}_{3}-10\right)$ ppm. MS (ESI) ([m/z], \%): $283.24(50), 371.24\left([\mathrm{M}+1]^{+}, 100\right)$. HRMS (ESI): 371.2434 calculated for $\mathrm{C}_{20} \mathrm{H}_{39} \mathrm{O}_{2} \mathrm{SSi}$ and found 371.2426.

Synthesis of $(((1 R, 3 S, Z)-5-(2-((4 \mathrm{a} S, 5 R, 6 R, 8 \mathrm{a} S, E)-4 \mathrm{a}, 6-$ dimethyl-5-((3-methyl-3-((trimethylsilyl)oxy)butyl)thio) octahydronaphthalen-1(2H)-ylidene)ethylidene)-4-methylenecyclohexane-1,3-diyl)bis(oxy))bis(tert-butyldimethylsilane) (12): $\mathrm{n}$-BuLi solution $2.5 \mathrm{M}$ in hexane was added dropwise to another solution of phospine oxide $9(347 \mathrm{mg}$, $0.595 \mathrm{mmol})$ in THF $(2 \mathrm{ml})$ at $-78^{\circ} \mathrm{C}$, and the deep red mixture was stirred for $1 \mathrm{~h}$. A solution of ketone $8(25 \mathrm{mg}$, $0.067 \mathrm{mmol})$ in THF $(1 \mathrm{~mL})$ was added at the same conditions via the cannula and stirred for $17 \mathrm{~h}$. A saturated aqueous solution of $\mathrm{NH}_{4} \mathrm{Cl}(5 \mathrm{~mL})$ was added, and the mixture was extracted with $\mathrm{CH}_{2} \mathrm{Cl}_{2}(3 \times 5 \mathrm{~mL})$. The organic phase was dried over $\mathrm{Na}_{2} \mathrm{SO}_{4}$ and filtered, and the solvent was removed under reduced pressure. The residue was purified by column chromatography with EtOAc/hexane (1\%) as the eluent, affording $12(27 \mathrm{mg}, 55 \%)$ as colourless oil; Rf: 0.95 (10\% EtOAc/hexane). IR (ATR, cm $\left.{ }^{-1}\right)$ : 2960, 2935, 2851, 1487, 1357, 1210, 1037, 852. $[\alpha]^{21}{ }_{\mathrm{D}}=+32.70(\mathrm{c}$ $\left.1.00, \mathrm{CHCl}_{3}\right) .{ }^{1} \mathrm{H}-\mathrm{NMR}\left(\mathrm{CDCl}_{3}, \delta\right): 6.25(1 \mathrm{H}, \mathrm{d}, J=11.0 \mathrm{~Hz}$, $\left.\mathrm{H}-8^{\prime \prime}\right), 6.02\left(1 \mathrm{H}, \mathrm{d}, J=11.0 \mathrm{~Hz}, \mathrm{H}-7^{\prime \prime}\right), 5.21(1 \mathrm{H}, \mathrm{d}, J=1.7 \mathrm{~Hz}$, $\left.\mathrm{H}_{2} \mathrm{C}=\mathrm{C}-4^{\prime \prime}\right), 4.88\left(1 \mathrm{H}, \mathrm{d}, J=2.0 \mathrm{~Hz}, \mathrm{H}_{2} \mathrm{C}=\mathrm{C}-4^{\prime \prime}\right), 4.39(1 \mathrm{H}, \mathrm{m}$, $\left.\mathrm{H}-3^{\prime \prime}\right), 4.21\left(1 \mathrm{H}, \mathrm{m}, \mathrm{H}-1^{\prime \prime}\right), 2.89(1 \mathrm{H}, \mathrm{d}, J=12.9 \mathrm{~Hz}, \mathrm{H}-5), 2.53$ $(3 \mathrm{H}, \mathrm{m}), 2.17(4 \mathrm{H}, \mathrm{m}), 1.81(7 \mathrm{H}, \mathrm{m}), 1.58(2 \mathrm{H}, \mathrm{m}), 1.41(2 \mathrm{H}$, $\mathrm{m}), 1.24\left(6 \mathrm{H}, \mathrm{s}, \mathrm{CH}_{3}-4^{\prime}\right), 1.22(2 \mathrm{H}, \mathrm{m}), 1.09(3 \mathrm{H}, \mathrm{d}$, $\left.J=6.4 \mathrm{~Hz}, \mathrm{CH}_{3}-9\right), 0.90\left(12 \mathrm{H}, \mathrm{s}, \mathrm{CH}_{3}{ }^{-}{ }^{t} \mathrm{Bu}\right), 0.89\left(6 \mathrm{H}, \mathrm{s}, \mathrm{CH}_{3^{-}}\right.$ $\left.{ }^{t} \mathrm{Bu}\right), 0.86$ (3H, s, $\left.\mathrm{CH}_{3}-10\right), 0.14$ (9H, s, $\left.\mathrm{CH}_{3}-\mathrm{Si} / \mathrm{TMS}\right), 0.09$ $\left(12 \mathrm{H}, \mathrm{s}, \mathrm{CH}_{3}-\mathrm{Si} / \mathrm{TBS}\right) \mathrm{ppm} .{ }^{13} \mathrm{C}-\mathrm{NMR}\left(\mathrm{CDCl}_{3}, \delta\right): 148.4(\mathrm{C}-$ $\left.4^{\prime \prime}\right), 142.4(\mathrm{C}-1), 135.1\left(\mathrm{C}-5^{\prime \prime}\right), 123.6\left(\mathrm{CH}-7^{\prime \prime}\right), 118.0(\mathrm{CH}-$ $\left.8^{\prime \prime}\right), 111.4\left(\mathrm{H}_{2} \mathrm{C}=\mathrm{C}-4^{\prime \prime}\right), 73.6\left(\mathrm{C}-3^{\prime}\right), 72.1\left(\mathrm{CH}-3^{\prime \prime}\right), 67.5(\mathrm{CH}-$ $\left.1^{\prime \prime}\right), 65.5(\mathrm{CH}-8 \mathrm{a}), 46.0\left(\mathrm{CH}_{2}\right), 45.1\left(\mathrm{CH}_{2}\right), 44.8\left(\mathrm{CH}_{2}\right), 44.3$ $(\mathrm{CH}), 43.0(\mathrm{C}-4 \mathrm{a}), 37.6\left(\mathrm{CH}_{2}\right), 37.6\left(\mathrm{CH}_{2}\right), 31.1(\mathrm{CH}), 30.8$ $\left(\mathrm{CH}_{2}\right), 30.2\left(\mathrm{CH}_{2}\right), 29.9\left(\mathrm{CH}_{3}-4^{\prime}\right), 29.9\left(\mathrm{CH}_{3}-4^{\prime}\right), 25.9\left(\mathrm{CH}_{3}-\right.$ $\left.{ }^{t} \mathrm{Bu}\right), 25.8\left(\mathrm{CH}_{3}{ }^{t} \mathrm{Bu}\right), 24.4\left(\mathrm{CH}_{2}\right), 23.4\left(\mathrm{CH}_{2}\right), 20.9\left(\mathrm{CH}_{3}-9\right)$, $18.8\left(\mathrm{CH}_{3}-10\right), 18.3\left(\mathrm{C}-{ }^{t} \mathrm{Bu}\right), 18.2\left(\mathrm{C}-{ }^{t} \mathrm{Bu}\right), 2.6\left(\mathrm{CH}_{3}-\mathrm{Si} /\right.$ TMS $),-4.6\left(\mathrm{CH}_{3}-\mathrm{Si} / \mathrm{TBS}\right),-4.7\left(\mathrm{CH}_{3}-\mathrm{Si} / \mathrm{TBS}\right),-4.8\left(\mathrm{CH}_{3}-\right.$ $\mathrm{Si} / \mathrm{TBS}),-5.1\left(\mathrm{CH}_{3}-\mathrm{Si} / \mathrm{TBS}\right)$ ppm. MS (ESI) ([m/z], \%): $735.50\left([\mathrm{M}+1]^{+}, 100\right), 757.48\left([\mathrm{M}+\mathrm{Na}]^{+}, 7\right)$. HRMS (ESI): 735.5058 calculated for $\mathrm{C}_{41} \mathrm{H}_{79} \mathrm{O}_{3} \mathrm{SSi}_{3}$ and found 735.5063 .

Synthesis of $(1 R, 3 S, Z)-5-(2-((4 \mathrm{a} S, 5 R, 6 R, 8 \mathrm{a} S, E)-5-((3-$ hydroxy-3-methylbutyl)thio)-4a,6-dimethyloctahydronaphthalen-1 $(2 H)$-ylidene)ethylidene)-4-methylenecyclohexane1,3-diol (7): TBAF 1.0M solution in THF $(0.120 \mathrm{ml}, 0.120$ $\mathrm{mmol})$ was added to another solution of $12(17 \mathrm{mg}, 0.023$ $\mathrm{mmol})$ in THF $(2 \mathrm{ml})$, and the mixture was stirred at r.t. for $24 \mathrm{~h}$. After that time, 2 equivalents of TBAF were added, and the mixture was stirred in these conditions for another $24 \mathrm{~h}$. Then, the reaction was concentrated under reduced pressure. The residue was purified by column chromatography with EtOAc/hexane (80\%) as the eluent, affording 7 (10 mg, 99\%) as colourless oil; Rf: 0.60 (EtOAc). IR (ATR, $\mathrm{cm}^{-1}$ ): 3367, $3318,2958,2937,2851,1490,1355,1208,1037 .[\alpha]$ ${ }_{\mathrm{D}}^{23}=+2.58\left(\mathrm{c} 1.00, \mathrm{CHCl}_{3}\right) \cdot{ }^{1} \mathrm{H}-\mathrm{NMR}\left(\mathrm{CDCl}_{3}, \delta\right): 6.38(1 \mathrm{H}$, d, $\left.J=11.1 \mathrm{~Hz}, \mathrm{H}-8^{\prime \prime}\right), 6.01\left(1 \mathrm{H}, \mathrm{d}, J=11.2 \mathrm{~Hz}, \mathrm{H}-7^{\prime \prime}\right), 5.34$ $\left(1 \mathrm{H}, \mathrm{d}, J=1.9 \mathrm{~Hz}, \mathrm{H}_{2} \mathrm{C}=\mathrm{C}-4^{\prime \prime}\right), 5.00(1 \mathrm{H}, \mathrm{d}, \mathrm{J}=2.0 \mathrm{~Hz}$, $\left.\mathrm{H}_{2} \mathrm{C}=\mathrm{C}-4^{\prime \prime}\right), 4.45\left(1 \mathrm{H}, \mathrm{dd}, J=7.6 / 4.4 \mathrm{~Hz}, \mathrm{H}-3^{\prime \prime}\right), 4.25(1 \mathrm{H}, \mathrm{m}$, $\left.\mathrm{H}-1^{\prime \prime}\right), 2.89(1 \mathrm{H}, \mathrm{m}), 2.59(4 \mathrm{H}, \mathrm{m}), 2.32(3 \mathrm{H}, \mathrm{m}), 2.05(4 \mathrm{H}$, $\mathrm{m}), 1.79(4 \mathrm{H}, \mathrm{m}), 1.57(1 \mathrm{H}, \mathrm{m}), 1.42(2 \mathrm{H}, \mathrm{m}), 1.26(6 \mathrm{H}, \mathrm{s}$, $\left.\mathrm{CH}_{3}-4^{\prime}\right), 1.19(1 \mathrm{H}, \mathrm{m}), 1.08\left(3 \mathrm{H}, \mathrm{d}, J=6.5 \mathrm{~Hz}, \mathrm{CH}_{3}-9\right), 0.89$ $(1 \mathrm{H}, \mathrm{m}), 0.87\left(3 \mathrm{H}, \mathrm{s}, \mathrm{CH}_{3}-10\right)$ ppm. ${ }^{13} \mathrm{C}-\mathrm{NMR}\left(\mathrm{CDCl}_{3}, \delta\right)$ : $147.6\left(\mathrm{C}-4^{\prime \prime}\right), 144.5(\mathrm{C}-1), 133.1\left(\mathrm{C}-5^{\prime \prime}\right), 125.4\left(\mathrm{CH}-7^{\prime \prime}\right), 117.3$ $\left(\mathrm{CH}-8^{\prime \prime}\right), 112.1\left(\mathrm{H}_{2} \mathrm{C}=\mathrm{C}-4^{\prime \prime}\right), 70.9\left(\mathrm{CH}-3^{\prime \prime}\right), 70.9\left(\mathrm{C}-3^{\prime}\right), 66.8$ $\left(\mathrm{CH}-1^{\prime \prime}\right), 66.1(\mathrm{CH}-8 \mathrm{a}), 45.3\left(\mathrm{CH}_{2}\right), 44.4(\mathrm{CH}), 43.6\left(\mathrm{CH}_{2}\right)$, $43.1(\mathrm{C}-4 \mathrm{a}), 42.8\left(\mathrm{CH}_{2}\right), 37.6\left(\mathrm{CH}_{2}\right), 31.1\left(\mathrm{CH}_{2}\right), 31.0(\mathrm{CH})$, $30.0\left(\mathrm{CH}_{2}\right), 29.9\left(\mathrm{CH}_{2}\right), 29.4\left(\mathrm{CH}_{3}-4^{\prime}\right), 29.3\left(\mathrm{CH}_{3}-4^{\prime}\right), 24.5$ $\left(\mathrm{CH}_{2}\right), 23.4\left(\mathrm{CH}_{2}\right), 20.9\left(\mathrm{CH}_{3}-9\right), 18.8\left(\mathrm{CH}_{3}-10\right)$ ppm. MS (ESI) $([\mathrm{m} / z], \%): 186.22(100), 435.29\left([\mathrm{M}+1]^{+}, 85\right), 457.27$ $\left([\mathrm{M}+\mathrm{Na}]^{+}, 83\right)$. HRMS (ESI): 435.2927 calculated for $\mathrm{C}_{26} \mathrm{H}_{43} \mathrm{O}_{3} \mathrm{~S}$ and found 435.2916 .

\section{Data Availability}

The data used to support the findings of this study are available in supplementary materials.

\section{Conflicts of Interest}

The authors declare that they have no conflicts of interest.

\section{Acknowledgments}

The work of the NMR, MS, and Unidade DRX Monocristal divisions of the research support services of the University of Vigo (CACTI) is gratefully acknowledged. This work was financially supported by the Xunta de Galicia (ED431C2017/ 70) and CITACA Strategic Partnership (ED431E2018/07).

\section{Supplementary Materials}

The supplementary data contain experimental procedures, characterization data, and copies of ${ }^{1} \mathrm{H}$ and ${ }^{13} \mathrm{C}$ spectra of all compounds. (Supplementary Materials)

\section{References}

[1] A. W. Norman, R. Bouillon, and M. Thomasset, Eds., Vitamin D: Chemistry, Biology and Clinical Applications of the Steroid Hormone, Vitamin D Workshop, Inc., Riverside, CA, USA, 1997.

[2] D. Feldman, F. H. Glorieux, and J. W. Pike, Vitamin D, Academic Press, San Diego, Ca, USA, 1997.

[3] R. Pardo and M. Santelli, "Synthesis of vitamin D metabolites. Review," Bulletin de la Societe Chimique de France, vol. 1, pp. 98-114, 1985.

[4] H. Dai and G. H. Posner, "Synthetic approaches to vitamin D," Synthesis, vol. 1994, no. 12, pp. 1383-1398, Article ID 25697, 1994.

[5] G.-D. Zhu and W. H. Okamura, "Synthesis of vitamin D (calciferol)," Chemical Reviews, vol. 95, no. 6, pp. 1877-1952, 1995.

[6] G. H. Posner and M. Kahraman, "Organic chemistry of vitamin D analogues (deltanoids)," European Journal of Organic Chemistry, vol. 2003, no. 20, pp. 3889-3895, Article ID 00264, 2003. 
[7] D. Feldman, J. W. Pike, and F. H. Glorieux, Vitamin D, Elsevier Academic Press, Burlington, MA, USA, 2nd edition, 2005.

[8] D. Feldman, W. Pike, and J. S. Adams, Vitamin D, Elsevier Academic Press, San Diego, CA, USA, 3rd edition, 2011.

[9] Y. Wu, S. Ling, C. D'Halleweyn et al., "Synthesis of CD-ring modified $1 \alpha, 25$-dihydroxy vitamin $\mathrm{D}$ analogs: five-membered D-ring analogs," Bioorganic \& Medicinal Chemistry Letters, vol. 7, pp. 923-928, 1997.

[10] A. Verstuyf, L. Verlinden, E. Van Etten et al., "Biological activity of CD-ring modified $1 \alpha, 25$-dihydroxyvitamin D analogs: C-ring and five-membered D-ring analogues," Journal of Bone and Mineral Research, vol. 15, pp. 237-252, 2000.

[11] G.-D. Zhu, Y. Chen, X. Zhou et al., "Synthesis of CD-ring modified $1 \alpha, 25$-dihydroxy vitamin $\mathrm{D}$ analogs: C-ring analogs," Bioorganic \& Medicinal Chemistry Letters, vol. 6, no. 14, pp. 1703-1708, 1996.

[12] X. Zhou, G.-D. Zhu, D. Van Haver et al., "Synthesis, biological activity, and conformational analysis of four seco-D-15,19bisnor- $1 \alpha, 25$-Dihydroxyvitamin $\mathrm{D}$ analogues, diastereomeric at C17 and C20," Journal of Medicinal Chemistry, vol. 42, no. 18 , pp. 3539-3556, 1999.

[13] B. Linclau, P. De Clercq, M. Vandewalle, R. Bouillon, and A. Verstuyf, "The synthesis of CD-ring modified $1 \alpha, 25$ dihydroxy vitamin $\mathrm{D}$ analogs: six-membered D-ring analogs. I," Bioorganic \& Medicinal Chemistry Letters, vol. 7, no. 11, pp. 1461-1464, 1997.

[14] S. Gabriëls, D. Van Haver, M. Vandewalle, P. De Clercq, A. Verstuyf, and R. Bouillon, "Development of analogues of $1 \alpha, 25$-dihydroxyvitamin D3 with biased side chain orientation: methylated des-C,D-homo analogues," Chemistry - A European Journal, vol. 7, pp. 520-532, 2001.

[15] G. Li, S. Kusari, and M. Spiteller, "Natural products containing 'decalin' motif in microorganisms," Natural Product Reports, vol. 31, no. 9, pp. 1175-1201, 2014.

[16] R. C. Jadulco, M. Koch, T. B. Kakule et al., "Isolation of pyrrolocins A-C: cis- and trans-decalin tetramic acid antibiotics from an endophytic fungal-derived pathway," Journal of Natural Products, vol. 77, no. 11, pp. 2537-2544, 2014.

[17] V. Singh, S. R. Iyer, and S. Pal, "Recent approaches towards synthesis of cis-decalins," Tetrahedron, vol. 61, no. 39, pp. 9197-9231, 2005.

[18] S. Okamoto, T. Hosoe, T. Itabashi et al., "New decalin derivatives, eujavanoic acids $\mathrm{A}$ and $\mathrm{B}$, from Eupenicillium javanicum," Journal of Natural Products, vol. 67, no. 9, pp. 1580-1583, 2004.

[19] T. Tokoroyama, "Synthesis of clerodane diterpenoids and related compounds - stereoselective construction of the decalin skeleton with multiple contiguous stereogenic centers," Synthesis, vol. 2000, no. 05, pp. 611-633, Article ID 6381, 2000.

[20] M. A. Varner and R. B. Grossman, "Annulation routes to trans-decalins,” Tetrahedron, vol. 55, no. 49, pp. 13867-13886, 1999.

[21] G. Mehta and V. Singh, "Progress in the construction of cyclooctanoid systems: new approaches and applications to natural product syntheses," Chemical Reviews, vol. 99, no. 3, pp. 881-930, 1999.

[22] G. Mehta and A. Srikrishna, "Synthesis of polyquinane natural products: an update," Chemical Reviews, vol. 97, no. 3, pp. 671-720, 1997.

[23] A. T. Merritt and S. V. Ley, "Clerodane diterpenoids," Natural Product Reports, vol. 9, no. 3, pp. 243-287, 1992.
[24] H. Santalla, O. N. Faza, G. Gómez, Y. Fall, and C. Silva López, "From hydrindane to decalin: a mild transformation through a dyotropic ring expansion," Organic Letters, vol. 19, no. 13, pp. 3648-3651, 2017.

[25] H. Santalla, Z. Gándara, U. Gómez-Bouzó, G. Gómez, and Y. Fall, "Easy access to polyfunctionalized chiral decalins," ACS Omega, vol. 5, no. 40, pp. 26049-26055, 2020.

[26] Y. Fall, O. Diouf, G. Gómez, and T. Bolaño, "The thioacetate approach to the synthesis of the side chain of vitamin D metabolites and analogues," Tetrahedron Letters, vol. 44, no. 32, pp. 6069-6072, 2003.

[27] Z. Gándara, O. Diouf, G. Gómez, and Y. Fall, "The thioacetate approach to vitamin D analogues. Part 2: synthesis of (25S)23-thia-1 $\alpha, 25,26$-trihydroxyvitamin D3," Tetrahedron Letters, vol. 48, no. 38, pp. 6735-6737, 2007.

[28] A. Mouriño, M. Torneiro, C. Vitale et al., "Efficient and versatile synthesis of A-ring precursors of 1i,25-dihydroxyvitamin D3 and analogs. Application to the synthesis of Lythgoe-Roche phosphine oxide," Tetrahedron Letters, vol. 38, pp. 4713-4716, 1997.

[29] A. R. Daniewski, L. M. Garofalo, S. D. Hutchings et al., "Efficient synthesis of the A-ring phosphine oxide building block useful for $1 \alpha, 25$-dihydroxy vitamin D3 and analogues," Journal of Organic Chemistry, vol. 67, no. 5, pp. 1580-1587, 2002. 